\title{
Impact of lowest instrumented vertebra tilt and rotation on uninstrumented lumbar curve and L4 tilt in thoracic adolescent idiopathic scoliosis
}

\author{
Akira Iwata, MD, PhD, ${ }^{1}$ Hideki Sudo, MD, PhD, ${ }^{1}$ Kuniyoshi Abumi, MD, PhD, ${ }^{2}$ Manabu Ito, MD, PhD, ${ }^{3}$ \\ Katsuhisa Yamada, MD, PhD, ${ }^{1}$ and Norimasa Iwasaki, MD, PhD'1 \\ 1Department of Orthopaedic Surgery, Hokkaido University Hospital; ${ }^{2}$ Spine Center, Sapporo Orthopaedic Hospital; and \\ ${ }^{3}$ Department of Spine and Spinal Cord Disorders, Hokkaido Medical Center, Sapporo, Hokkaido, Japan
}

\begin{abstract}
OBJECTIVE Controversy exists regarding the effects of lowest instrumented vertebra (LIV) tilt and rotation on uninstrumented lumbar segments in adolescent idiopathic scoliosis (AIS) surgery. Because the intraoperative LIV tilt from the inferior endplate of the LIV to the superior sacral endplate is not stable after surgery, the authors measured the LIV angle of the instrumented thoracic spine as the LIV angle of the construct. This study aimed to evaluate the effects of the LIV angle of the construct and the effects of LIV rotation on the postoperative uninstrumented lumbar curve and L4 tilt in patients with thoracic AIS.
\end{abstract}

METHODS A retrospective correlation and multivariate analysis of a prospectively collected, consecutive, nonrandomized series of patients at a single institution was undertaken. Eighty consecutive patients with Lenke type 1 or type 2 AIS treated with posterior correction and fusion were included. Preoperative and 2-year postoperative radiographic measurements were the outcome measures for this study. Outcome variables were postoperative uninstrumented lumbar segments (LIV tilt, LIV translation, uninstrumented lumbar curve, thoracolumbar/lumbar [TL/L] apical vertebral translation $[\mathrm{AVT}]$, and L4 tilt). The LIV angle of the construct was measured from the orthogonal line drawn from the upper instrumented vertebra to the LIV. Multiple stepwise linear regression analysis was conducted between outcome variables and patient demographics/radiographic measurements. There were no study-specific biases related to conflicts of interest.

RESULTS Predictor variables for postoperative uninstrumented lumbar curve were the postoperative LIV angle of the construct, number of uninstrumented lumbar segments, and flexibility of TL/L curve. Specifically, a lower postoperative uninstrumented lumbar curve was predicted by a lower absolute value of the postoperative LIV angle of the construct ( $p$ $<0.0001$ ). Predictor variables for postoperative L4 tilt were postoperative LIV rotation, preoperative L4 tilt, and preoperative uninstrumented lumbar curve. Specifically, a lower postoperative L4 tilt was predicted by a lower absolute value of postoperative LIV rotation $(p<0.0001)$.

CONCLUSIONS The LIV angle of the construct significantly affected the LIV tilt, uninstrumented lumbar curve, and TL/L AVT. LIV rotation significantly affected the LIV translation and L4 tilt.

https://thejns.org/doi/abs/10.3171/2020.4.SPINE191297

KEYWORDS adolescent idiopathic scoliosis; thoracic curve; posterior spinal correction and fusion; lowest instrumented vertebra; vertebral rotation; L4 tilt; lumbar; deformity

$\mathrm{S}$ ELECTION of the lowest instrumented vertebra (LIV) in thoracic adolescent idiopathic scoliosis (AIS) is an important factor in preventing distal lumbar coronal curve decompensation and/or L4 tilt. Some studies that aimed to identify the appropriate LIV level on preoperative radiographs ${ }^{1-6}$ demonstrated that a neutral and stable vertebra was the ideal LIV for preventing distal lumbar coronal curve decompensation., ${ }^{3,4}$ In Lenke type 1A curves, surgeons may need to obtain maximum reduction of the apical translation of the main thoracic curve and to extend the LIV, at least to the last vertebra touching the central sacral vertical line (CSVL), in order to avoid postoperative distal lumbar coronal curve decompensation. ${ }^{5}$

Even after selecting the correct LIV, decompensation can still occur, ${ }^{8}$ indicating that other important factors, such as LIV tilt or LIV rotational angle (LIV rotation), should be

ABBREVIATIONS AIS = adolescent idiopathic scoliosis; AVT = apical vertebral translation; CSVL = central sacral vertical line; LIV = lowest instrumented vertebra; SRS-22 = Scoliosis Research Society-22 questionnaire; TL/L = thoracolumbar/lumbar; UEV = upper end vertebra.

SUBMITTED October 30, 2019. ACCEPTED April 9, 2020.

INCLUDE WHEN CITING Published online June 12, 2020; DOI: 10.3171/2020.4.SPINE191297. 
considered. As for LIV tilt, a horizontal LIV may theoretically restore the vertebra to a normal anatomical position, which allows for better correction of the lumbar curve.? Alternatively, a tilted LIV may align more harmoniously with the unfused vertebra immediately inferior to it, which may prevent decompensation. ${ }^{7}$ However, there have been few studies on the effect of postoperative LIV tilt or rotation on the radiographic outcome measurements of posterior thoracic fusion in AIS. ${ }^{1,7-9}$ In particular, no studies have examined the effect of LIV rotation. Because the intraoperative LIV tilt from the inferior endplate of the LIV to the superior sacral endplate is not stable after surgery, ${ }^{1}$ we measured the LIV angle of the instrumented thoracic spine as the LIV angle of the construct. We hypothesize that maintaining the target LIV angle of the construct and LIV rotation would help control the uninstrumented lumbar segments. This study aimed to evaluate the effects of the LIV angle of the construct and LIV rotation on the uninstrumented lumbar segments in patients with thoracic AIS.

\section{Methods}

After institutional review board approval, data from 80 consecutive patients (6 males and 74 females) who underwent posterior thoracic curve correction for Lenke $1(\mathrm{n}=$ 59) or Lenke $2(\mathrm{n}=21)$ AIS (lumbar modifier A, $\mathrm{n}=49$; B, $\mathrm{n}=17$; and $\mathrm{C}, \mathrm{n}=14$ ) at our institution between June 2008 and March 2015 were retrospectively collected.

All patients had a main thoracic Cobb angle of $\leq 90^{\circ}$. The exclusion criteria included Lenke type 3-6 AIS curves and scoliosis of the syndromic, neuromuscular, and congenital types. No cases were lost to follow-up. The mean age and Risser sign at surgery were 15.1 years (range 9-23 years) and 3.6 (range 0-5), respectively. The mean body mass index at surgery was $18.6 \mathrm{~kg} / \mathrm{m}^{2}$ (range $12-25 \mathrm{~kg} / \mathrm{m}^{2}$ ).

In all patients, standing long-cassette posteroanterior radiographs were evaluated for multiple parameters before surgery and on follow-up after 2 years. Curve flexibility was assessed using supine lateral passive bending radiographs. Coronal Cobb measurements of the proximal thoracic curve, main thoracic curve, thoracolumbar/lumbar $(\mathrm{TL} / \mathrm{L})$ curve, and the uninstrumented lumbar curve were obtained. The end vertebral levels in the main thoracic curve were determined on preoperative radiographs and measured on subsequent radiographs to maintain consistency for statistical comparisons. Sagittal measurements included thoracic kyphosis (T5-12), TL/L kyphosis (T10L2), and lumbar lordosis (L1-S1). Coronal balance was measured by lateral displacement of the $\mathrm{C} 7$ coronal plumb line from the CSVL (C7-CSVL). Sagittal balance was evaluated by measuring the absolute displacement value of the $\mathrm{C} 7$ plumb line relative to the $\mathrm{S} 1$ posterior superior corner as the sagittal vertical axis. LIV translation and $\mathrm{TL} / \mathrm{L}$ apical vertebral translation (AVT) were measured as the distance between the geometrical center of the LIV and the apical vertebra and CSVL, respectively.

The tilting angle of the LIV or the L4 vertebra (i.e., LIV tilt or L4 tilt) was measured as the inclination of the inferior endplate of the vertebra from the horizontal plane. LIV tilt and L4 tilt were defined as positive ("+") or negative ("-") when the preoperative opening was concave or convex, respectively. The preoperative LIV tilt and LIV angle of the construct were measured in accordance with the vertebra for the postoperative LIV tilt and LIV angle of the construct, respectively. In addition, the LIV angle of the construct was measured from the orthogonal line drawn from the upper instrumented vertebra to the LIV (Fig. 1) and was defined as positive ("+") or negative (" -") when its preoperative opening against the orthogonal line drawn from the upper instrumented vertebra was concave or convex, respectively. In our hospital, postoperative CT scans were routinely obtained for clinical reasons, such as confirmation of pedicle screw malposition, not for the purposes of this study.

The LIV rotation angle was measured on CT scans before and immediately after surgery. ${ }^{10-12}$ The angles of vertebral rotation were measured with reference to the junction of the laminae, the dorsal central aspect of the vertebral foramen, the middle of the vertebral body, and the sagittal plane. ${ }^{10-12}$ LIV rotation was defined as positive $(+)$ or negative $(-)$ when the rotation relative to the pelvis was clockwise or counterclockwise, respectively.

For measurements, two board-certified orthopedic specialists with more than 15 years of experience independently assessed the radiographs and CT scans. Our internal studies on inter- and intrarater reliability have shown excellent kappa statistics for all continuous measures (range 0.90-0.99).

The pre- and postoperative clinical outcomes at the 2-year follow-up were evaluated using the Scoliosis Research Society-22 questionnaire (SRS-22).

\section{Surgical Technique}

The fusion level selection was based on both standing and bending radiographs. The bending radiographs were obtained from supine anteroposterior passive bending radiographs. The instrumentation levels were determined from end-to-end vertebrae on standing radiographs in most cases, and vertebrae without rotation on the bending films were selected as the LIV, ${ }^{10,12,13}$ except for the following cases: when the distance between the geometrical center of the upper end vertebra (UEV) and the C7 plumb line was $>20 \mathrm{~mm}$, a UIV one level above the UEV was selected regardless of shoulder balance for the Lenke 1 curves. When the UEV was T1 for the Lenke 2 curves, T2 was selected as the UIV. In addition, regarding the LIV selection for both Lenke 1 and 2 curves, when the LEV was not located at a stable vertebra, the LIV was determined to be one or two levels distal to the LEV until the LIV was located at a stable vertebra.

Surgeries were performed, as described previously. ${ }^{10,12}$ Briefly, after exposure of the posterior spinal elements, pedicle screws (USS II, DePuy Spine) were placed. After connecting the two rods to all the screw heads, the two rods were simultaneously rotated..$^{10,12}$ Distraction force was first applied on each screw head on the concave side of the thoracic curve, so that both the scoliosis and thoracic kyphosis could be corrected more effectively by lengthening the posterior column..$^{10,12}$ Then, compression force was applied segmentally on the convex curve. ${ }^{10,12}$ An in situ rod-bending maneuver was not conducted during the 
surgery ${ }^{10,12}$ Local bone grafting followed decortication of the laminae. A brace was not required in any patient.

\section{Statistical Analysis}

Data are expressed as the mean \pm standard deviation for continuous values. Independent-samples t-tests were used to compare between-group differences in demographic data. Pearson's correlation coefficient analyses were used to assess the relationships between two continuous data. Multiple stepwise linear regression analysis was conducted to control for possible confounding variables..$^{13}$ Outcome variables were postoperative uninstrumented lumbar segments (LIV tilt, LIV translation, uninstrumented lumbar curve, TL/L AVT, and L4 tilt) that were considered as independent variables. The following covariates were tested in the multivariate regression analysis as patients' preoperative background status: age at surgery, Risser sign, body mass index, preoperative proximal thoracic curve, main thoracic curve, TL/L curve, flexibility of main thoracic and TL/L curve, LIV angle of the construct, LIV rotation, uninstrumented lumbar curve, L4 tilt, level of thoracic apical vertebra, thoracic kyphosis, TL/L kyphosis, lumbar lordosis, C7-CSVL, sagittal vertebral axis, and TL/L AVT. In addition, the postoperative LIV angle of the construct, LIV rotation, and number of uninstrumented lumbar segments were also tested because they are intraoperatively controllable variates. Significant multivariate predictors of uninstrumented lumbar segments are reported with their respective predictive equations, including the intercept and regression coefficients $(\beta) .{ }^{13}$ Model fit was assessed by the goodness-of-fit $\mathrm{F}$ test and $\mathrm{R}^{2}$ statistic. ${ }^{13}$ Data analysis was performed using JMP statistical software (version 13; SAS, Inc.). Any p value < 0.05 was considered statistically significant.

\section{Results}

The demographic characteristics of the patients are summarized in Table 1. There were no significant undesired outcomes such as in instrumented curve progression or loss of balance that required revision surgery.

\section{Independent Outcomes Within the Uninstrumented Lumbar Segments}

Postoperative uninstrumented lumbar curve and L4 tilt were the independent outcomes $\left(\mathrm{R}^{2}=0.055\right)$. The other outcomes such as postoperative LIV tilt (goodness-of-fit $\mathrm{F}$ test $=47.209, \mathrm{R}^{2}=0.551, \mathrm{p}<0.0001$ ), LIV translation (goodness-of-fit $\mathrm{F}$ test $=40.025, \mathrm{R}^{2}=0.665, \mathrm{p}<0.0001$ ), and TL/L AVT (goodness-of-fit F test $=41.128, \mathrm{R}^{2}=0.516$, $\mathrm{p}<0.0001$ ) were dependent on the postoperative uninstrumented lumbar curve and L4 tilt.

\section{Predictors of Postoperative Uninstrumented Lumbar Curve and L4 Tilt}

Multiple stepwise linear regression analysis indicated that 3 variables were independent predictors of postoperative uninstrumented lumbar curve: postoperative LIV angle of the construct, number of uninstrumented lumbar segments, and flexibility of the TL/L curve. The model fit the data well (goodness-of-fit $\mathrm{F}$ test $=48.280, \mathrm{R}^{2}=0.656$,

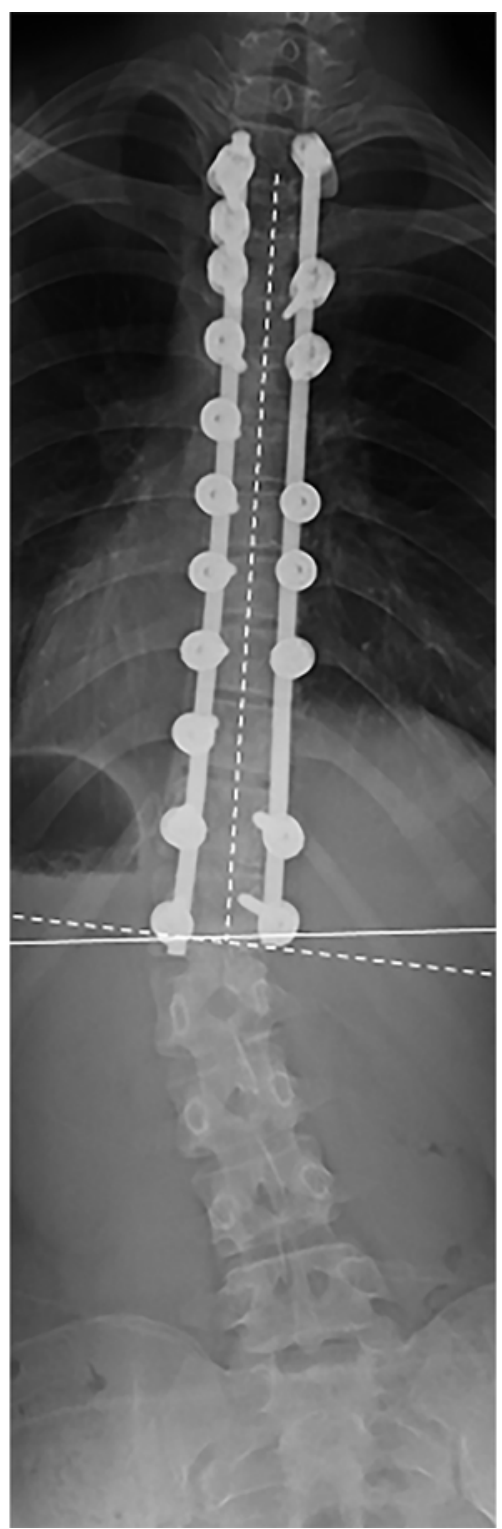

FIG. 1. The LIV angle of the construct is measured from the orthogonal line drawn from the upper instrumented vertebra to the LIV.

$\mathrm{p}<0.001$ ), indicating that $66 \%$ of uninstrumented lumbar curves were explained by the 3 significant independent predictors (Table 2). Specifically, lower postoperative uninstrumented lumbar curve was predicted by a lower absolute value of postoperative LIV angle of the construct $(\mathrm{p}<0.0001)$.

Multiple stepwise linear regression analysis also indicated that 3 variables were independent predictors of postoperative L4 tilt: postoperative LIV rotation, preoperative L4 tilt, and preoperative uninstrumented lumbar curve. The model fit the data well (goodness-of-fit $\mathrm{F}$ test $=49.333, \mathrm{R}^{2}=0.661, \mathrm{p}<0.0001$ ), indicating that $66 \%$ of postoperative L4 tilts were explained by the 3 significant independent predictors (Table 3). Specifically, lower postoperative L4 tilt was predicted by a lower absolute value of postoperative LIV rotation ( $\mathrm{p}<0.0001$ ). 


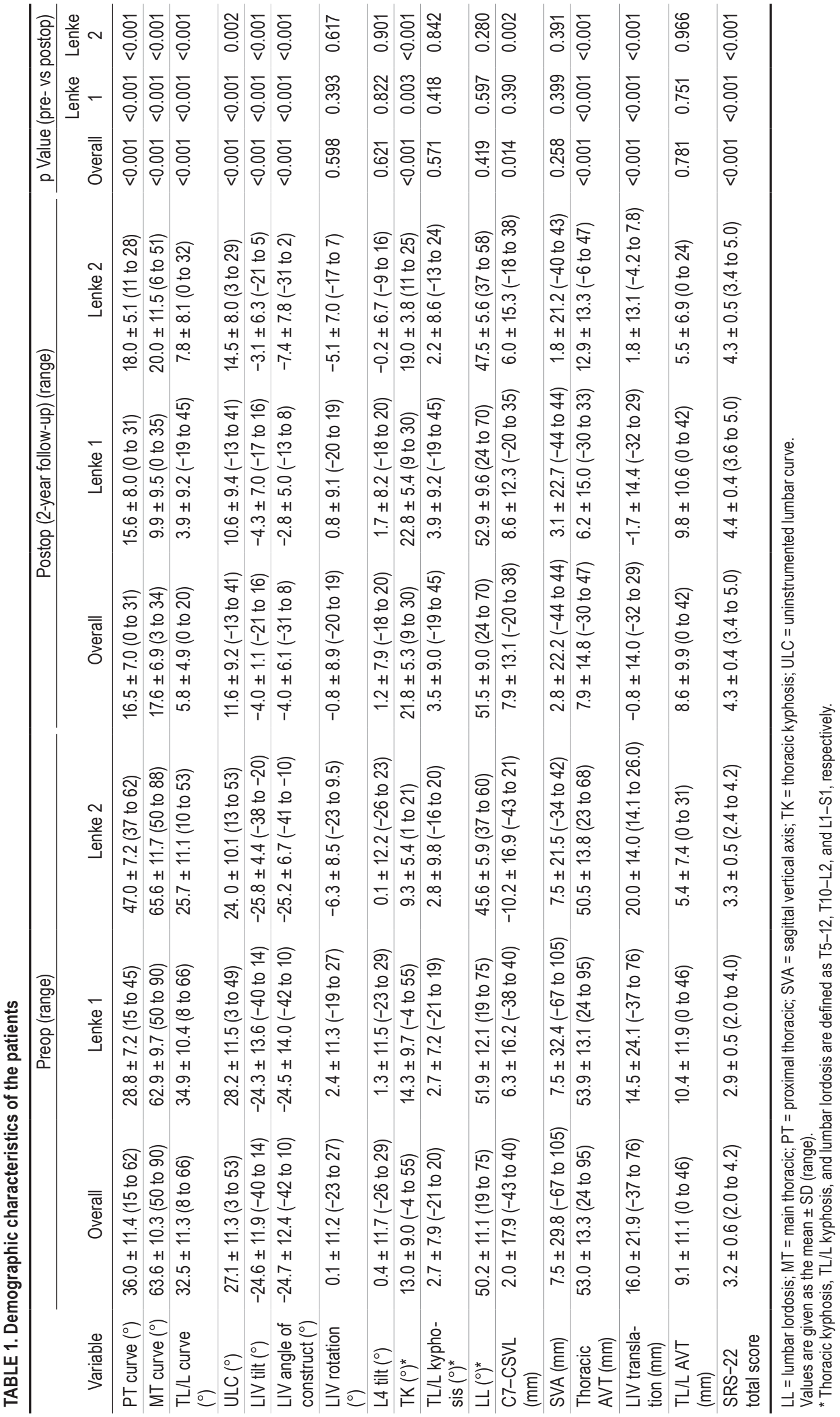


TABLE 2. Predictors for uninstrumented lumbar curve using multiple stepwise linear regression analysis

\begin{tabular}{lcccrrr}
\hline \multicolumn{1}{c}{ Variable } & $\begin{array}{c}\text { Regression } \\
\text { Coefficient }(\beta)\end{array}$ & $\begin{array}{c}\text { Standard } \\
\text { Error }\end{array}$ & $95 \% \mathrm{Cl}$ & $\mathrm{F}$ & Standardized $\beta$ & $\mathrm{p}$ Value \\
\hline Constant & 1.497 & 3.444 & $(-5.252,8.247)$ & & 11.625 & $<0.001$ \\
\hline Postop LIV angle of construct $\left(^{\circ}\right)$ & -0.689 & 0.117 & $(-0.918,-0.458)$ & 34.418 & -37.447 & $<0.001$ \\
\hline No. of uninstrumented lumbar segments & 2.970 & 0.602 & $(1.789,4.151)$ & 24.301 & 30.857 & $<0.001$ \\
\hline Flexibility of TL/L curve $(\%)$ & -5.951 & 1.736 & $(-9.354,-2.548)$ & 11.750 & -19.337 & $<0.001$ \\
\hline
\end{tabular}

\section{Effects of Postoperative LIV Angle of the Construct and LIV Rotation on the Uninstrumented Lumbar Segments}

With regard to the uninstrumented lumbar segments, there was little association between LIV angle of the construct and LIV rotation $\left(\mathrm{R}^{2}=0.125\right)$. Multiple regression analysis showed that the postoperative LIV angle of the construct combined with the preoperative value of uninstrumented lumbar segments correlated with postoperative uninstrumented lumbar curve (goodness-of-fit $\mathrm{F}$ test $\left.=46.566, \mathrm{R}^{2}=0.547, \mathrm{p}<0.0001\right)$, LIV tilt (goodness-of-fit $\mathrm{F}$ test $\left.=41.620, \mathrm{R}^{2}=0.519, \mathrm{p}<0.0001\right)$, and TL/L AVT (goodness-of-fit $\mathrm{F}$ test $=35.329, \mathrm{R}^{2}=0.479, \mathrm{p}=0.0001$ ) (Table 4).

The analysis also showed that postoperative LIV rotation combined with the preoperative value of uninstrumented lumbar segments correlated with postoperative L4 tilt (goodness-of-fit F test $=60.200, \mathrm{R}^{2}=0.610, \mathrm{p}<0.0001$ ) and LIV translation (goodness-of-fit $\mathrm{F}$ test $=60.404, \mathrm{R}^{2}=$ 0.611, p < 0.0001) (Table 4).

\section{Separate Analysis of the Lenke 1 and Lenke 2 Curves to Determine the Impact of the Upper Thoracic Curve on the Uninstrumented Lumbar Curve and L4 Tilt}

The multiple regression analysis did not show a significant correlation between postoperative proximal thoracic curve and uninstrumented lumbar curve $(p=0.260)$. The correlation coefficients between postoperative proximal thoracic curve and uninstrumented lumbar curve were $0.065(\mathrm{p}=0.735)$ for Lenke type 1 curves and $0.184(\mathrm{p}$ $=0.429$ ) for Lenke type 2 curves, respectively. Similarly, multiple regression analysis did not show a significant correlation between postoperative proximal thoracic curve and L4 tilt $(\mathrm{p}=0.469)$. The correlation coefficient between postoperative proximal thoracic curve and L4 tilt was $-0.191(p=0.295)$ for Lenke type 1 curves and 0.327 ( $p=0.148)$ for Lenke type 2 curves, respectively.

The multiple regression analysis showed a significant correlation between the LIV angle of the construct and uninstrumented lumbar curve in both Lenke type 1 ( $\mathrm{p}=$ $0.0001)$ and Lenke type $2(\mathrm{p}<0.0001)$ curves. Likewise, the multiple regression analysis showed a significant correlation between postoperative LIV rotation and L4 tilt in both Lenke type $1(\mathrm{p}<0.0001)$ and Lenke type $2(\mathrm{p}=$ 0.006) curves.

\section{Correlation Between the Uninstrumented Lumbar Segments and SRS-22 Outcome Scores}

The correlation coefficient between the preoperative uninstrumented lumbar curve and SRS-22 total scores was $0.320(p=0.097)$ (for Lenke 1 curves, it was -0.167 $[\mathrm{p}=0.360]$, and for Lenke 2 curves, it was -0.115 [p = $0.621])$. At the 2-year postoperative follow-up, the correlation coefficient was $-0.135(p=0.420)$ (for Lenke 1 curves, it was 0.065 [ $p=0.735]$, and for Lenke 2 curves, it was 0.184 [p $=0.429]$ ). The correlation coefficient between the preoperative L4 tilt and SRS-22 total score was 0.202 ( $p=0.304$ ) (for Lenke 1 curves, it was -0.167 [ $=0.360]$, and for Lenke 2 curves, it was -0.115 [p = 0.621]). At the 2-year postoperative follow-up, the correlation coefficient was 0.078 ( $\mathrm{p}=0.645$ ) (for Lenke 1 curves, it was 0.065 [p $=0.735]$, and for Lenke 2 curves, it was $0.184[p=0.429]$ ). The correlations between other radiographic parameters and SRS-22 outcome scores are shown in Table 5.

\section{Case Presentation}

A 16-year-old female AIS patient with Lenke type $1 \mathrm{CN}$ (lumbar modifier $\mathrm{C}$ and normal thoracic kyphosis) underwent posterior spinal fusion at T4-L1 (Fig. 2). The flexibility of the TL/L curve was $93 \%$. The postoperative uninstrumented lumbar segments approximated the expected values.

\section{Discussion}

The current results showed that an uninstrumented lumbar curve and L4 tilt were independent outcome vari-

TABLE 3. Predictors of $L 4$ tilt using multiple stepwise linear regression analysis

\begin{tabular}{lcccrrr}
\hline \multicolumn{1}{c}{ Variable } & $\begin{array}{c}\text { Regression } \\
\text { Coefficient }(\beta)\end{array}$ & Standard Error & $95 \% \mathrm{Cl}$ & $\mathrm{F}$ & Standardized $\beta$ & $p$ Value \\
\hline Constant & -3.364 & 1.410 & $(-6.127,-0.601)$ & & 1.169 & 0.017 \\
\hline Postop LIV rotation $\left(^{\circ}\right)$ & 0.374 & 0.069 & $(0.239,0.509)$ & 29.571 & 29.642 & $<0.001$ \\
\hline Preop ULC lumbar $\left(^{\circ}\right)$ & 0.165 & 0.049 & $(0.069,0.261)$ & 11.375 & 16.447 & 0.001 \\
\hline Preop L4 tilt $\left(^{\circ}\right)$ & 0.363 & 0.051 & $(0.264,0.462)$ & 51.287 & 37.577 & $<0.001$ \\
\hline
\end{tabular}


TABLE 4. Effects of postoperative LIV angle of the construct and LIV rotation on the uninstrumented lumbar segments using multiple stepwise linear regression analysis

\begin{tabular}{|c|c|c|c|c|c|c|}
\hline Variable & $\begin{array}{c}\text { Regression } \\
\text { Coefficient }(\beta)\end{array}$ & Standard Error & $95 \% \mathrm{Cl}$ & $\mathrm{F}$ & Standardized $\beta$ & $\mathrm{p}$ Value \\
\hline \multicolumn{7}{|l|}{ Postop ULC $\left(^{\circ}\right)$} \\
\hline Constant & 2.127 & 1.877 & $(-1.552,5.806)$ & & 11.625 & 0.257 \\
\hline Preop data & 0.225 & 0.071 & $(0.085,0.365)$ & 9.992 & 2.4222 & 0.021 \\
\hline LIV angle of construct $\left({ }^{\circ}\right)$ & -0.851 & 0.130 & $(-1.106,-0.595)$ & 42.562 & -46.278 & $<0.001$ \\
\hline \multicolumn{7}{|l|}{ Postop LIV tilt $\left(^{\circ}\right)$} \\
\hline Constant & 2.341 & 1.245 & $(-0.100,4.782)$ & & -3.988 & 0.06 \\
\hline Preop data & 0.155 & 0.050 & $(0.057,0.253)$ & 9.602 & 16.336 & 0.002 \\
\hline LIV angle of construct $\left({ }^{\circ}\right)$ & 0.629 & 0.097 & $(0.439,0.819)$ & 42.097 & 34.205 & $<0.001$ \\
\hline \multicolumn{7}{|l|}{ Postop L4 tilt $\left({ }^{\circ}\right)$} \\
\hline Constant & 1.043 & 0.564 & $(-0.062,2.148)$ & & 1.17 & 0.064 \\
\hline Preop data & 0.377 & 0.054 & $(0.272,0.483)$ & 49.193 & 39.064 & $<0.001$ \\
\hline LIV rotation $\left(^{\circ}\right)$ & 0.308 & 0.070 & $(0.171,0.446)$ & 19.261 & 24.444 & $<0.001$ \\
\hline \multicolumn{7}{|l|}{ Postop LIV translation (mm) } \\
\hline Constant & -6.586 & 1.309 & $(-9.151,-4.021)$ & & -0.813 & $<0.001$ \\
\hline Preop data & 0.335 & 0.057 & $(0.223,0.447)$ & 34.276 & 65.361 & $<0.001$ \\
\hline LIV rotation $\left({ }^{\circ}\right)$ & -0.541 & 0.141 & $(-0.817,-0.265)$ & 14.743 & -42.866 & $<0.001$ \\
\hline \multicolumn{7}{|l|}{ Postop TL/L AVT (mm) } \\
\hline Constant & 1.730 & 1.261 & $(-0.741,4.202)$ & & 8.638 & 0.170 \\
\hline Preop data & 0.633 & 0.075 & $(0.485,0.781)$ & 70.583 & 62.453 & $<0.001$ \\
\hline LIV angle of construct $\left({ }^{\circ}\right)$ & -0.287 & 0.137 & $(-0.554,-0.019)$ & 4.397 & -15.588 & 0.036 \\
\hline
\end{tabular}

ables. The other outcomes, such as postoperative LIV tilt, LIV translation, and TL/L AVT, were dependent on the postoperative uninstrumented lumbar curve and L4 tilt. The LIV angle of the construct was the primary factor that affects the uninstrumented lumbar curve. In addition, LIV rotation was the primary factor that affects L4 tilt. The postoperative LIV angle of the construct contributed to
$55 \%$ of uninstrumented lumbar curves, $52 \%$ of LIV tilts, and $48 \%$ of TL/L AVTs. In addition, postoperative LIV rotation contributed to $61 \%$ of L4 tilts and $61 \%$ of LIV translations. The proximal thoracic curve did not correlate with uninstrumented lumbar segments.

In this study, the regression coefficient between the postoperative uninstrumented lumbar curve and the flex-

TABLE 5. Correlation coefficient between radiographic parameters and SRS-22 scores

\begin{tabular}{lrrrrr}
\hline \multirow{2}{*}{ Variable } & \multicolumn{5}{c}{ Correlation Coefficient (p value) } \\
\cline { 2 - 6 } & LIV Tilt $\left({ }^{\circ}\right)$ & LIV Translation $(\mathrm{mm})$ & ULC $\left(^{\circ}\right)$ & \multicolumn{1}{c}{ TL/L AVT $(\mathrm{mm})$} & L4 Tilt $\left({ }^{\circ}\right)$ \\
\hline Preop & & & & \\
\hline Function/activity & $-0.072(0.716)$ & $0.112(0.572)$ & $0.321(0.096)$ & $-0.218(0.266)$ & $0.251(0.198)$ \\
\hline Pain & $-0.161(0.412)$ & $0.064(0.746)$ & $0.279(0.151)$ & $-0.273(0.161)$ & $0.181(0.357)$ \\
\hline Self-image/appearance & $0.131(0.507)$ & $-0.003(0.988)$ & $0.105(0.596)$ & $0.104(0.598)$ & $0.129(0.514)$ \\
\hline Mental health & $-0.263(0.168)$ & $0.136(0.482)$ & $0.270(0.156)$ & $-0.184(0.339)$ & $0.120(0.534)$ \\
\hline Satisfaction & $0.006(0.976)$ & $-0.097(0.625)$ & $0.225(0.249)$ & $0.175(0.372)$ & $0.060(0.763)$ \\
\hline Total & $0.198(0.312)$ & $-0.073(0.714)$ & $0.320(0.097)$ & $0.009(0.965)$ & $0.202(0.304)$ \\
\hline Postop (2-year follow-up) & & & & & \\
\hline Function/activity & $-0.296(0.071)$ & $-0.162(0.332)$ & $0.085(0.612)$ & $0.325(0.046)$ & $0.207(0.212)$ \\
\hline Pain & $-0.062(0.712)$ & $-0.023(0.883)$ & $0.002(0.976)$ & $0.289(0.078)$ & $0.124(0.457)$ \\
\hline Self-image/appearance & $0.100(0.054)$ & $-0.124(0.457)$ & $-0.212(0.202)$ & $0.050(0.766)$ & $0.107(0.522)$ \\
\hline Mental health & $-0.009(0.956)$ & $-0.024(0.887)$ & $-0.137(0.413)$ & $0.210(0.207)$ & $-0.092(0.583)$ \\
\hline Satisfaction & $0.189(0.257)$ & $-0.003(0.984)$ & $-0.201(0.227)$ & $0.035(0.836)$ & $-0.120(0.474)$ \\
\hline Total & $0.039(0.816)$ & $-0.104(0.534)$ & $-0.135(0.420)$ & $0.260(0.115)$ & $0.078(0.645)$ \\
\hline
\end{tabular}




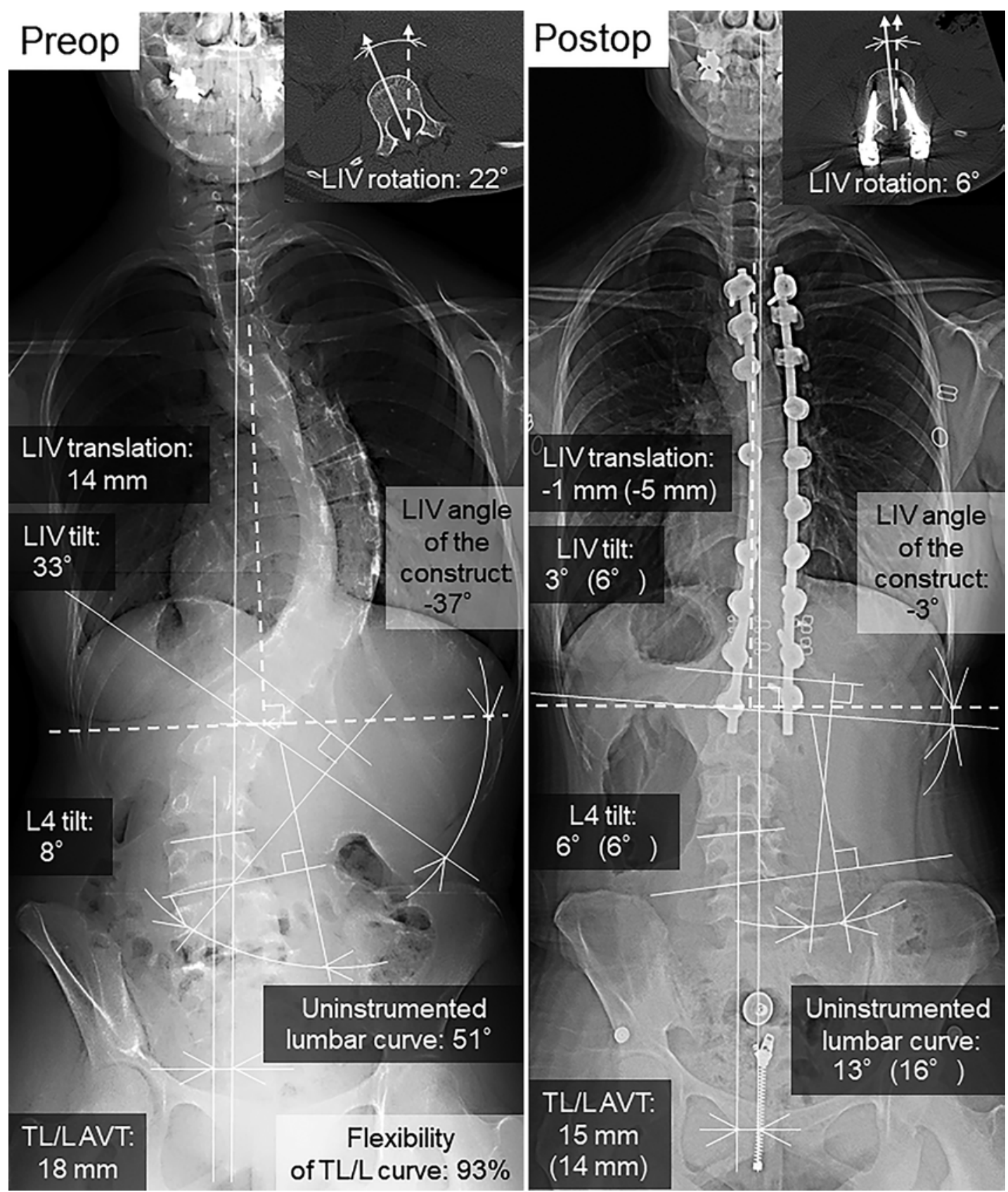

FIG. 2. A 16-year-old adolescent girl with idiopathic scoliosis with a Lenke type $1 \mathrm{CN}$ (lumbar modifier $\mathrm{C}$ and normal thoracic kyphosis) curve. Values in parentheses reflect the expected value.

ibility of the TL/L curve seemed to be large, indicating that higher flexibility can enable greater lumbar curve correction. In addition, although the number of uninstrumented lumbar segments had a significant positive correlation with the Cobb angles of the uninstrumented lumbar curve, the number of uninstrumented lumbar segments did not correlate with L4 tilt. These results indicate that extension of the instrumentation to the caudal level can likely correct the uninstrumented lumbar curve; however, it cannot correct the L4 tilt.

Few studies have examined the relationship between LIV tilt and radiographic outcome measurements. ${ }^{7,9}$
Ishikawa et al. ${ }^{9}$ documented that spontaneous correction of the TL/L curves occurred consistently by correction of the main thoracic curve and by making the LIV more horizontal after posterior thoracic fusion for the Lenke type 1 and 2 curves. These results may have been because LIV tilt is multifactorially dependent on other radiographic parameters.

We measured the LIV angle of the instrumented thoracic spine as the LIV angle of the construct. Surgeons can confirm and handle the angle during application of compression/distraction forces to the screws, even after rod rotation. To evaluate the LIV angle of the construct intra- 


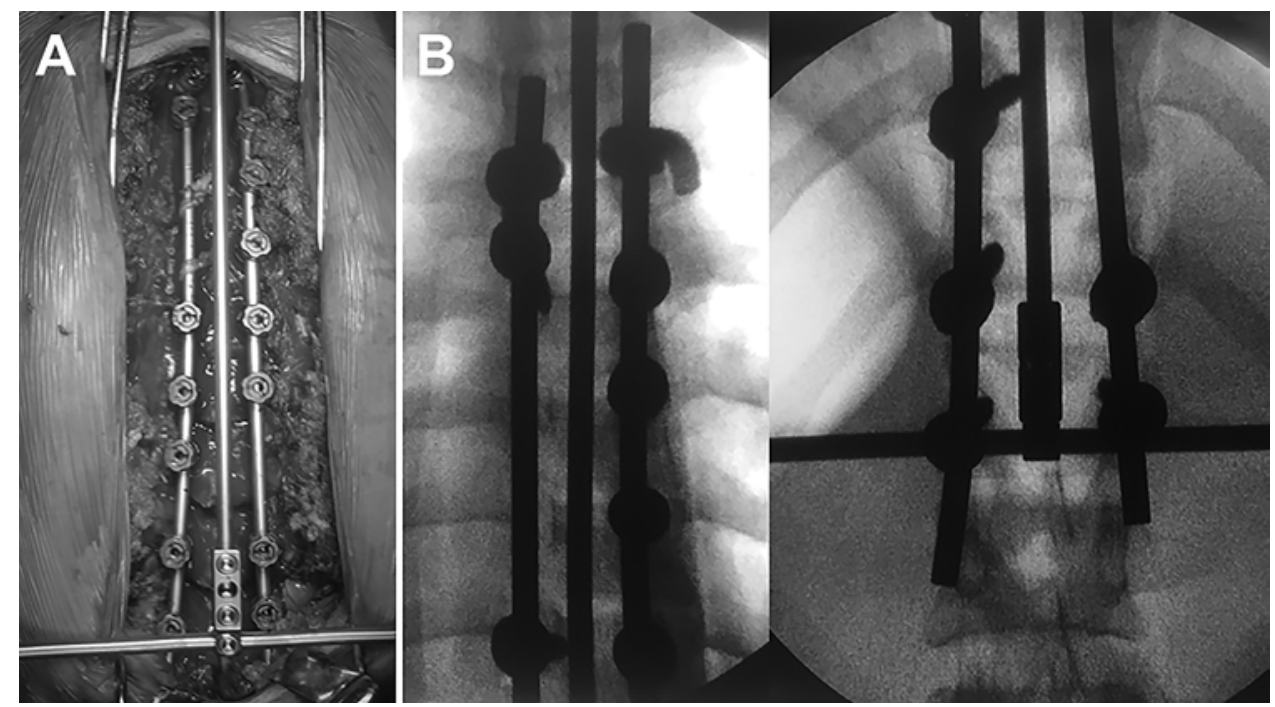

FIG. 3. A custom-made T-shaped metallic bar (A) and fluoroscopic images (B) for intraoperative evaluation of the LIV angle of the construct.

operatively, a custom-made T-shaped metallic bar and/or fluoroscopy was used (Fig. 3). In this study, the LIV angle of the construct significantly correlated with, and may be substituted for, the LIV tilt.

In this study, the LIV angle of the construct significantly affected the uninstrumented lumbar curve, and LIV rotation significantly affected the L4 tilt. First, the uninstrumented lumbar curve significantly correlated with the LIV angle of the construct, indicating that correction of the uninstrumented lumbar curve mainly depended on the correction of the LIV angle of the construct. Second, L4 tilt significantly correlated with LIV rotation, suggesting that LIV rotation should be corrected for horizontalization of L4. When taken together, in both Lenke type 1 and 2 curves, LIV tilt into the lumbar curve would create a more harmonious overall spinal alignment and specifically help prevent postoperative decompensation in the frontal plane. ${ }^{7}$ Because the prevalence of the adding-on phenomenon is similar between Lenke $1(15 \%)$ and Lenke $2(12 \%)$ curves,${ }^{14}$ the current results could be useful for both Lenke type 1 and 2 curves.

Because LIV rotation can significantly affect the L4 tilt, it would be ideal for the LIV to become a neutral vertebra after surgery. The use of monoaxial pedicle screws and the single-rod rotation maneuver may enable easy control of the rotation. On the other hand, simultaneous double-rod rotation can induce de-rotation of the apical vertebra and restore thoracic kyphosis, and does not intend to manipulate vertebral rotation at each level separately. ${ }^{10,12}$ Therefore, the medical device in this study that uses the polyaxial pedicle screw may have significant limitations for the correction of LIV rotation.

In this study, the SRS-22 scores showed no significant correlation with uninstrumented lumbar segments. There has been only one report investigating LIV tilt and other radiographic measurements with SRS-22 outcomes. ${ }^{15} \mathrm{Al}-$ though there was no significant association between the 10-year composite radiographic score and SRS-22 scores, disc wedging subjacent to the LIV $\left(\geq 5^{\circ}\right)$ led to significant disc degeneration below the LIV.$^{15}$ For longer periods, this disc degeneration may affect the patient reported outcomes. Although the follow-up period of this study was only 2 years, coronal balance was reported to be stable over 20 years if the uninstrumented lumbar curve was corrected to $<35^{\circ} .{ }^{16}$ Meanwhile, the L4 tilt should be $<15^{\circ}$ to prevent curve progression and low-back pain. ${ }^{17}$ These results indicate that the target angle for postoperative uninstrumented lumbar curve $\left(<35^{\circ}\right)$ and L4 tilt $\left(<15^{\circ}\right)$ should be achieved to prevent curve progression.

\section{Study Limitations}

This study has some limitations. First, the CT-measured LIV rotation with patients in the supine position could have changed in the standing position. However, AlAubaidi et al. ${ }^{18}$ compared the precision of 3D geometry using the EOS imaging system with $\mathrm{CT}$ and reported that the results obtained using EOS imaging are comparable to those obtained using CT. Second, the present study has a limited number of patients in the sample, albeit consecutive in nature. Given the number of variables included in the model, we would have required approximately 200 patients to ensure the model is not overfit. ${ }^{13}$ Given that we had only 80 patients, this study is underpowered to translate these findings to clinical practice..$^{13}$

\section{Conclusions}

The angle measured from the orthogonal line drawn from the upper instrumented vertebra to the LIV (i.e., LIV angle of the construct) significantly affected the LIV tilt, uninstrumented lumbar curve, and TL/L AVT, while LIV rotation significantly affected the LIV translation and L4 tilt.

\section{References}

1. Barsi J, Caprio B, Garg S, et al. Do intraoperative LIV-tilt 
and disk angle remain stable at 2-year follow-up compared with upright radiographs in patients with idiopathic scoliosis? A retrospective cohort study. J Spinal Disord Tech. 2015;28(7):264-269.

2. Bridwell KH. Selection of instrumentation and fusion levels for scoliosis: where to start and where to stop. Invited submission from the Joint Section Meeting on Disorders of the Spine and Peripheral Nerves, March 2004. J Neurosurg Spine. 2004;1(1):1-8.

3. Lenke LG, Betz RR, Bridwell KH, et al. Spontaneous lumbar curve coronal correction after selective anterior or posterior thoracic fusion in adolescent idiopathic scoliosis. Spine (Phila Pa 1976). 1999;24(16):1663-1672.

4. Luk KD, Don AS, Chong CS, et al. Selection of fusion levels in adolescent idiopathic scoliosis using fulcrum bending prediction: a prospective study. Spine (Phila Pa 1976). 2008;33(20):2192-2198.

5. Matsumoto M, Watanabe K, Hosogane N, et al. Postoperative distal adding-on and related factors in Lenke type 1A curve. Spine (Phila Pa 1976). 2013;38(9):737-744.

6. Suk SI, Lee SM, Chung ER, et al. Determination of distal fusion level with segmental pedicle screw fixation in single thoracic idiopathic scoliosis. Spine (Phila Pa 1976). 2003;28(5):484-491.

7. Skaggs DL, Seehausen DA, Yamaguchi KT Jr, et al. Assessment of lowest instrumented vertebra tilt on radiographic measurements in Lenke "C" modifier curves undergoing selective thoracic fusion in adolescent idiopathic scoliosis. Spine Deform. 2016;4(2):125-130.

8. Cho W, Faloon MJ, Essig D, et al. Additional risk factors for adding-on after selective thoracic fusion in adolescent idiopathic scoliosis: implication of lowest instrumented vertebra angle and lumbosacral takeoff. Spine Deform. 2018;6(2):164169.

9. Ishikawa M, Cao K, Pang L, et al. Postoperative behavior of thoracolumbar/lumbar curve and coronal balance after posterior thoracic fusion for Lenke $1 \mathrm{C}$ and $2 \mathrm{C}$ adolescent idiopathic scoliosis. J Orthop Sci. 2015;20(1):31-37.

10. Sudo H, Abe Y, Abumi K, et al. Surgical treatment of double thoracic adolescent idiopathic scoliosis with a rigid proximal thoracic curve. Eur Spine J. 2016;25(2):569-577.

11. Sudo H, Abe Y, Kokabu T, et al. Impact of multilevel facetectomy and rod curvature on anatomical spinal reconstruction in thoracic adolescent idiopathic scoliosis. Spine (Phila Pa 1976). 2018;43(19):E1135-E1142.

12. Sudo H, Ito M, Abe Y, et al. Surgical treatment of Lenke 1 thoracic adolescent idiopathic scoliosis with maintenance of kyphosis using the simultaneous double-rod rotation technique. Spine (Phila Pa 1976). 2014;39(14):1163-1169.
13. Sudo H, Abe Y, Kokabu T, et al. Correlation analysis between change in thoracic kyphosis and multilevel facetectomy and screw density in main thoracic adolescent idiopathic scoliosis surgery. Spine J. 2016;16(9):1049-1054.

14. Yang M, Zhao Y, Yin X, et al. Prevalence, risk factors, and characteristics of the "adding-on" phenomenon in idiopathic scoliosis after correction surgery: a systematic review and meta-analysis. Spine (Phila Pa 1976). 2018;43(11):780-790.

15. Lonner BS, Ren Y, Upasani VV, et al. Disc degeneration in unfused caudal motion segments ten years following surgery for adolescent idiopathic scoliosis. Spine Deform. 2018;6(6):684-690.

16. Larson AN, Fletcher ND, Daniel C, Richards BS. Lumbar curve is stable after selective thoracic fusion for adolescent idiopathic scoliosis: a 20-year follow-up. Spine (Phila Pa 1976). 2012;37(10):833-839.

17. Ohashi M, Watanabe K, Hirano T, et al. Predicting factors at skeletal maturity for curve progression and low back pain in adult patients treated nonoperatively for adolescent idiopathic scoliosis with thoracolumbar/lumbar curves: a mean 25-year follow-up. Spine (Phila Pa 1976). 2018;43(23):E1403-E1411.

18. Al-Aubaidi Z, Lebel D, Oudjhane K, Zeller R. Threedimensional imaging of the spine using the EOS system: is it reliable? A comparative study using computed tomography imaging. J Pediatr Orthop B. 2013;22(5):409-412.

\section{Disclosures}

The authors report no conflict of interest concerning the materials or methods used in this study or the findings specified in this paper.

\section{Author Contributions}

Conception and design: Sudo, Iwata. Acquisition of data: Sudo, Iwata. Analysis and interpretation of data: Sudo. Drafting the article: Sudo, Iwata. Critically revising the article: Sudo. Reviewed submitted version of manuscript: Sudo, Iwata, Yamada. Approved the final version of the manuscript on behalf of all authors: Sudo. Statistical analysis: Iwata. Administrative/technical/material support: Abumi, Ito, Yamada. Study supervision: Abumi, Ito, Iwasaki.

\section{Correspondence}

Hideki Sudo: Hokkaido University, Hokkaido, Japan. hidekisudo@yahoo.co.jp. 